\title{
Perinatal Citalopram Exposure Selectively Increases Locus Ceruleus Circuit Function in Male Rats
}

\author{
Ryan D. Darling, ${ }^{1}$ Loai Alzghoul, ${ }^{2}$ Junlin Zhang, ${ }^{1}$ Nidhi Khatri, ${ }^{3}$ Ian A. Paul, ${ }^{3,4}$ Kimberly L. Simpson, ${ }^{1,4}$ \\ and Rick C. S. Lin ${ }^{1,4}$ \\ ${ }^{1}$ Department of Neurobiology and Anatomical Sciences, ${ }^{2}$ Program in Neuroscience, and Departments of ${ }^{3}$ Pharmacology and Toxicology and ${ }^{4}$ Psychiatry \\ and Human Behavior, University of Mississippi Medical Center, Jackson, Mississippi 39216
}

Selective serotonin reuptake inhibitors (SSRIs), such as citalopram (CTM), have been widely prescribed for major depressive disorder, not only for adult populations, but also for children and pregnant mothers. Recent evidence suggests that chronic SSRI exposure in adults increases serotonin (5-HT) levels in the raphe system and decreases norepinephrine (NE) locus ceruleus (LC) neural activity, suggesting a robust opposing interaction between these two monoamines. In contrast, perinatal SSRI exposure induces a long-lasting downregulation of the 5-HT-raphe system, which is opposite to that seen with chronic adult treatment. Therefore, the goal of the present investigation was to test the hypothesis that perinatal CTM exposure $(20 \mathrm{mg} / \mathrm{kg} / \mathrm{d})$ from postnatal day 1 (PN1) to PN10 leads to hyperexcited NE-LC circuit function in adult rats ( $>$ PN90). Our single-neuron LC electrophysiological data demonstrated an increase in spontaneous and stimulus-driven neural activity, including an increase in phasic bursts in CTM-exposed animals. In addition, we demonstrated a corresponding immunoreactive increase in the rate-limiting catalyzing catecholamine enzyme (tyrosine hydroxylase) within the LC and their neocortical target sites compared to saline controls. Moreover, these effects were only evident in male exposed rats, suggesting a sexual dimorphism in neural development after SSRI exposure. Together, these results indicate that administration of SSRIs during a sensitive period of brain development results in long-lasting alterations in NE-LC circuit function in adults and may be useful in understanding the etiology of pervasive developmental disorders such as autism spectrum disorder.

\section{Introduction}

The pathophysiology underlying major depressive disorder remains poorly understood; however, selective serotonin reuptake inhibitors (SSRIs), including citalopram (CTM), have been widely prescribed and are preferred due to their low toxicity and wide therapeutic index. So far, most of our knowledge regarding the effects of antidepressant treatment has been obtained from studies of adult human or rodent populations. A major conclusion derived from these studies is that such exposure not only upregulates the 5-HT-raphe system, but also downregulates the noradrenergic locus ceruleus (NE-LC) system (Nestler et al., 1990; Szabo et al., 1999; West et al., 2009). One of the potential mechanisms behind such opposite effects on these two interconnected modulatory systems (Cedarbaum and Aghajanian, 1978; Luppi et al., 1995; Kim et al., 2004) is that 5-HT inhibits LC function (McRae-Degueurce et al., 1982; Bobker and Williams, 1989; Haddjeri et al., 1997).

In addition to their traditional roles in adults, 5- $\mathrm{HT}$ and $\mathrm{NE}$ are also known to play critical roles in neurodevelopment (Gas-

\footnotetext{
Received July 21, 2011; revised Aug. 31, 2011; accepted Sept. 6, 2011.

Author contributions: R.D.D., I.A.P., K.L.S., and R.C.S.L. designed research; R.D.D., L.A., J.Z., and N.K. performed research; R.D.D. analyzed data; R.D.D. and R.C.S.L. wrote the paper.

This research was supported by NIH EUREKA Grant MH084194 (R.C.S.L.).

Correspondence should be addressed to Dr. Rick C. S. Lin, Departments of Neurobiology and Anatomical Sciences and Psychiatry and Human Behavior, University of Mississippi Medical Center, Jackson, MS 39216. E-mail: rlin@umc.edu.

DOI:10.1523/JNEUROSCI.3736-11.2011

Copyright $\odot 2011$ the authors $\quad 0270-6474 / 11 / 3116709-07 \$ 15.00 / 0$
}

par et al., 2003; Sanders et al., 2005), and these roles may be sex specific (Connell et al., 2004). Early antidepressant exposure in male rats results in long-lasting behavioral effects, as well as a reduction in expression of the 5-HT synthetic enzyme (tryptophan hydroxylase) within the raphe nuclear complex and of the 5-HT transporter (SERT) within their cortical efferent fibers (Mirmiran et al., 1981; Maciag et al., 2006; Oberlander et al., 2009; Iñiguez et al., 2010; Weaver et al., 2010; Rodriguez-Porcel et al., 2011). In a recent study of adolescent [postnatal day 45 (PN45)] rats exposed to a SSRI, West et al. (2010) reported that brief ( 2 or $4 \mathrm{~d}$ ) treatment increased LC neuronal activity, suggesting an opposite effect compared to adult treatment. It is still not clear how SSRI exposure during early brain development affects NE-LC function, but it appears that it is different from adult exposure and that brief exposure can have dramatic effects that are observed well into adulthood.

At present, prescription of SSRIs to children and pregnant mothers is considered relatively safe (Cohen, 2007; KendallTackett and Hale, 2010), but adverse biological consequences of such early exposure are suspected (Casper et al., 2003; Hendrick et al., 2003; Moses-Kolko et al., 2005; Homberg et al., 2010), including a suspected role in autism spectrum disorder (ASD) (Chugani et al., 1999; Chandana et al., 2005; Whitaker-Azmitia, 2005; Croen et al., 2011). Interestingly, ASD is approximately four times more prevalent in boys, suggesting a sexual dimorphism that may be related to abnormal monoamine levels during early brain development. Therefore, the goal of the present investigation was to explore the sex-specific electrophysiological and 
immunohistochemical consequences of perinatal SSRI exposure on adult NE-LC circuit function.

\section{Materials and Methods}

Animals and drug application. Offspring from four timed-pregnant LongEvans rats purchased from Harlan Laboratories were cross-fostered on PN1 to achieve groups of 12-14 per litter. Each litter included ( $~ 3)$ pups from each treatment group, and no offspring were lost. A total of 21 Long-Evans rat pups (male $=11$, female $=10$ ) were used in this study who were injected subcutaneously $(2 \times / \mathrm{d})$ from PN1 to PN10 with either CTM $(n=12)(10 \mathrm{mg} / \mathrm{kg})$ (Toronto Research Chemicals) or saline $(n=$ 9) as described previously (Maciag et al., 2006). The dosing schedule (2 injections/d) was chosen to allow for a more dilute concentration of drug per injection to minimize the risk of injury at the injection site. The dose was selected to approximate the upper range of maternal and placental serum reported in clinical reports of maternal antidepressant treatment (Maciag et al., 2006). Maternal and offspring behavior were not overtly affected by cross-fostering or injections of either saline or CTM to pups, and we continue to examine behavioral effects associated with our drug exposure (Maciag et al., 2006; Rodriguez-Porcel et al., 2011). At PN28, pups were weaned and housed in groups of $2-3$ per cage under standard laboratory conditions with ad libitum access to food and water. When animals reached adulthood ( $>90 \mathrm{~d}$ ), they were then used for electrophysiological and immunohistochemical studies. All experimental animal procedures were approved by the University of Mississippi Medical Center Institutional Animal Care and Use Committee.

Electrophysiological recording. Animals were anesthetized with sodium pentobarbital $(50 \mathrm{mg} / \mathrm{kg}$, i.p.) and positioned in a stereotaxic apparatus with bregma $3.0 \mathrm{~mm}$ ventral to lambda. This position of the animal's head helps to increase successful location of the LC along its long axis as well as to decrease the likelihood of penetrating the sinus rostral to the cerebellum. The coordinates for LC (given this head position) were 3.5$3.9 \mathrm{~mm}$ caudal to lambda and $1.0-1.2 \mathrm{~mm}$ lateral to midline (Paxinos and Watson, 1986). Standard electrophysiological criteria for identifying single LC neurons were considered as described previously (Aston-Jones and Bloom, 1981; Berridge and Waterhouse, 2003). Either tungsten (1.0-3.0 M $\Omega$; FHC) or bundled (eight stainless steel) microwire arrays (0.3-1.0 M $\Omega$; Neurobiological Laboratory) were lowered into the LC using a Kopf micropositioner. Neuronal signals were acquired and digitized using Plexon hardware (MAP) and software (Sort Client 2.60). After stable recordings were acquired, baseline recordings were made for $\sim 10$ min followed by tail-pinch recordings for $\sim 5 \mathrm{~min}$, which included $\sim 1 \mathrm{~s}$ tail pinch every $30 \mathrm{~s}$ throughout the recording session. Such compression reliably elicits significant increases of LC firing and shows little or no sensitization or habituation with repeated trials. Following experiments, electrode locations were marked using a 15 s $75-150 \mu \mathrm{A}$ DC current injection for histological verification.

Digitized unit recordings were further spike separated with Offline Sorter version 2.88 (Plexon) using various waveform characteristics including peak, valley, and principal components. Defined single units were exported to NeuroExplorer version 3.266 (NEX Technologies) for further analyses. Rate histograms were created using the baseline recordings to calculate spontaneous activity of LC neurons and averaged to determine the mean spike rate. Perievent interspike interval rasters with histograms were created using the tail pinch recordings to visualize event-related responses. The median interspike interval following the tail pinch was used to quantify the stimulus-driven discharge rates across groups. Stimulus-elicited bursting was quantified using the NeuroExplorer burst analysis function with the following parameters: $0.1 \mathrm{~s}$ maximum interval to start burst, $0.1 \mathrm{~s}$ maximum interval to end burst, $0.2 \mathrm{~s}$ minimum interval between burst, $0.1 \mathrm{~s}$ minimum duration of burst, and a minimum of two spikes in burst. These correspond to typical criteria used to quantify bursts in the LC (Seager et al., 2004; Devilbiss and Waterhouse, 2011). Baseline spike-rate averages, median interspike intervals, and burst analyses following tail pinch were statistically analyzed using $2 \times 2$ ANOVAs and post hoc simple main effects tests (SPSS version 18).

Immunohistochemistry. After the electrophysiological recordings, animals were perfused through the ascending aorta with $0.9 \%$ saline fol- lowed by $3.5 \%$ paraformaldehyde. Brains were sectioned with an $\mathrm{AO}$ freezing microtome at $40 \mu \mathrm{m}$. Tissues were processed in sets so that at least one member of each group was processed simultaneously as a set to avoid confounds in staining procedures. Sections from the LC region and neocortex were collected and processed using an anti-rabbit tyrosine hydroxylase (TH) antibody (1:1000 dilution; Millipore) for $48 \mathrm{~h}$ at $4^{\circ} \mathrm{C}$. The tissue was then incubated in a biotinylated anti-rabbit IgG (BA9200, ABC Kit, Vector Laboratories) for $1 \mathrm{~h}$, rinsed in PBS, and then linked with Cy2-conjugated streptavidin (1:200 dilution, Jackson ImmunoResearch Laboratories) for $1 \mathrm{~h}$ at room temperature. To determine whether $\mathrm{TH}$-immunoreactive fibers in the neocortex are noradrenergic in origin, double immunofluorescent methods were conducted. For double immunostaining, brain tissues were processed first for TH immunoreactivity as described, and then for either antimouse dopamine- $\beta$-hydroxylase (DBH; 1:1000 dilution; Millipore) or anti-mouse norepinephrine transporter (NET) antibody (1:1000 dilution; Mab Co) for $48 \mathrm{~h}$ at $4^{\circ} \mathrm{C}$. The neural profiles were then visualized using anti-mouse Cy3 (1:200 dilutions) for $1 \mathrm{~h}$ at room temperature.

To determine changes in the density and intensity of $\mathrm{TH}$ and $\mathrm{DBH} /$ NET immunoreactivity attributable to SSRI exposure, standard analyses were performed as described previously (Maciag et al., 2006; Weaver et al., 2010; Zhang et al., 2011). Briefly, digital photomicrographs of sections containing LC neurons and neocortex (especially the somatosensory cortex) were taken with consistent exposure times at 10 or 20 magnification, respectively, using a Nikon E800 epifluorescent microscope equipped with SenSys cool camera (Roper Scientific). Images were analyzed with MetaMorph Imaging software (Molecular Devices; version 6.2r6) using a threshold analysis to determine the average density and intensity of immunostaining within (1) all usable LC soma regions (typically only contralateral to recording sites) and (2) three cortical columns of each hemisphere of neocortex. Values within animals were averaged and then compared within staining sets to determine a percentage increase/decrease in staining by comparing experimental animals to their corresponding saline controls. Statistical analysis was done using one-sample $t$ tests (SPSS version 18).

\section{Results}

\section{Hyperexcitability of single LC neurons}

CTM-exposed male rats demonstrated approximately threefold increase in spontaneous LC firing rate (Fig. $1 A, B$ ) with a significant sex by drug exposure interaction $\left(F_{(1,83)}=4.279, p=\right.$ 0.042). Post hoc analysis revealed a simple main effect of drug exposure for males $\left(F_{(1,83)}=5.732, p=0.019\right)$. While spike sorting individual LC neurons, careful observation revealed a qualitative difference in the spike width of individual LC neurons between male rats exposed to CTM and saline (Fig. 1C). There was a significant sex by drug exposure interaction of spike width $\left(F_{(1,120)}=19.946, p<0.001\right)$, and a post hoc test revealed a simple main effect of drug exposure for males $\left(F_{(1,120)}=38.181, p<\right.$ $0.001)$. This suggests a change in ion channel conductances during the action potential, but would need to be investigated further with more precise (intracellular) recording techniques. Furthermore, there was a hyperexcitability following tail-pinch in CTMexposed male rats compared to saline-exposed male rats (Fig. $2 A, B)$, with a significant sex by drug exposure interaction $\left(F_{(1,108)}=8.831, p=0.004\right)$ and a simple main effect of drug exposure for males $\left(F_{(1,108)}=14.052, p<0.001\right)$. Individual LC neurons are known to operate in distinct modes of tonic and phasic firing (for review, see Berridge and Waterhouse, 2003; Aston-Jones and Cohen, 2005). We found that the phasic bursting response to tail pinch of CTM-exposed male rats also demonstrated a statistically significant interaction between sex and drug exposure in terms of bursts $/ \mathrm{min}\left(F_{(1,108)}=6.821, p=\right.$ $0.010)$, percentage spikes in a burst $\left(F_{(1,108)}=20.394, p<0.001\right)$, mean burst duration $\left(F_{(1,108)}=11.084, p=0.001\right)$, and mean spikes in a burst $\left(F_{(1,108)}=18.939, p<0.000\right)$ (Fig. $\left.2 C\right)$. Post hoc 
A
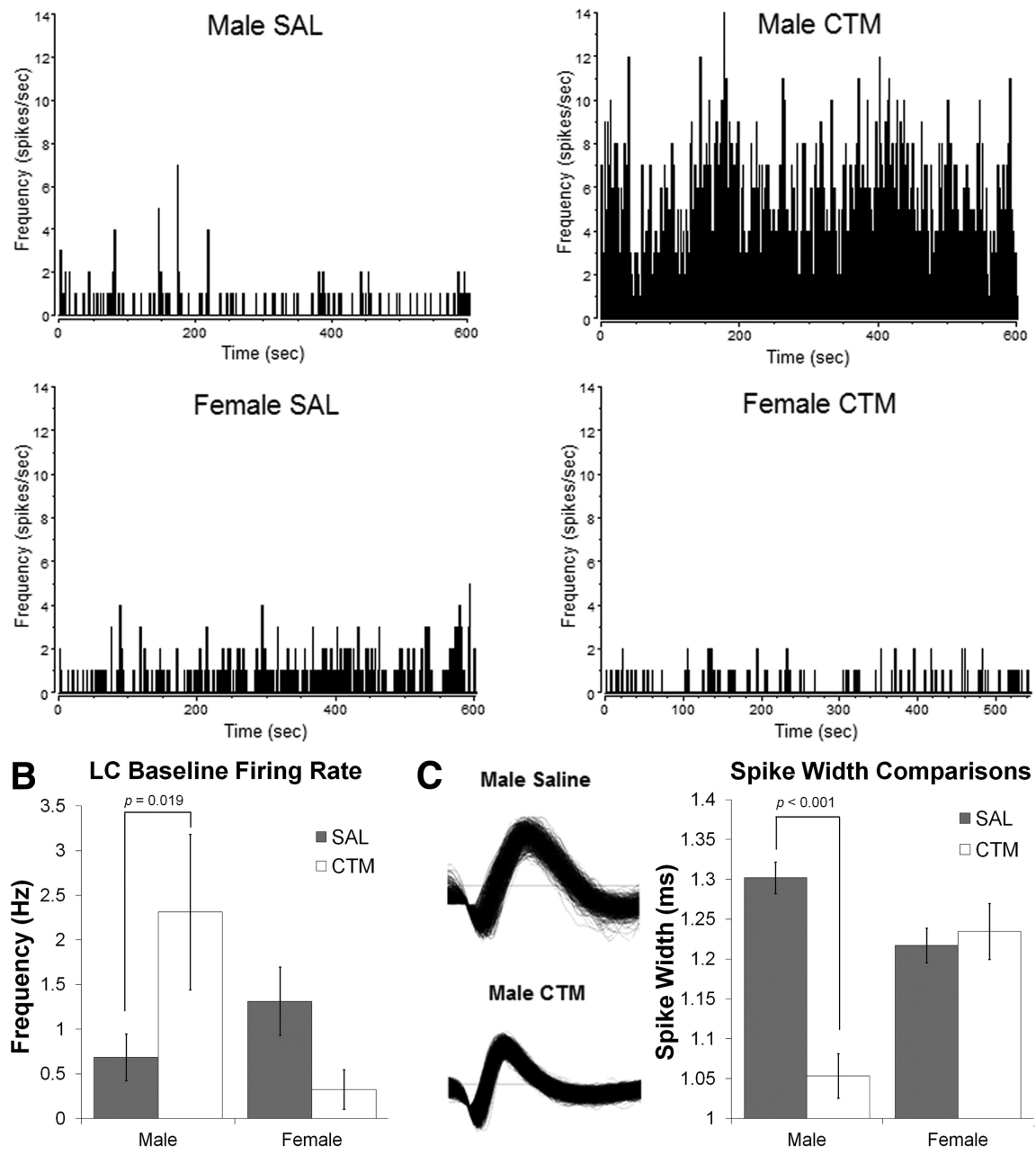

Figure 1. Spontaneous firing rates and waveform characteristics of single $L C$ neurons. $A, B$, Spontaneous firing rates of single $L C$ neurons were higher in male rats neonatally exposed to CTM $(2.31 \mathrm{~Hz} \pm 4.08 \mathrm{SD} ; n=22)$ compared to male saline (SAL) $(0.69$ $\mathrm{Hz} \pm 1.55 \mathrm{SD} ; n=34)$, female SAL $(1.31 \mathrm{~Hz} \pm 1.87 \mathrm{SD} ; n=24)$, and female $\mathrm{CTM}(0.33 \pm 0.58 \mathrm{SD} ; n=7)$ animals, verified with a significant sex by drug exposure interaction $\left(F_{(1,83)}=4.28, p=0.042\right)$ and a simple main effect of drug exposure for males $\left(F_{(1,83)}=5.732, p=0.019\right)$. Baseline measurements were taken over $\sim 10 \mathrm{~min}$ after stable recordings were acquired. Histograms are of representative animals over $\sim 10 \mathrm{~min}$ and are in $1 \mathrm{~s}$ bins. $C$, Single $L C$ neuron waveforms were narrow in male rats exposed to CTM ( $1.05 \pm 0.11 \mathrm{~ms} \mathrm{SD} ; n=15)$ compared to male SAL ( $1.30 \pm 0.15 \mathrm{~ms} \mathrm{SD} ; n=55)$, female SAL ( $1.22 \pm 0.14 \mathrm{~ms} \mathrm{SD} ; n=$ $41)$, and female CTM (1.23 $\pm 0.13 \mathrm{~ms} S D ; n=13)$ animals, suggesting a possible alteration in ion channel function. A sex by drug exposure interaction was significant $\left(F_{(1,120)}=19.95, p<0.001\right)$ with a simple main effect of drug exposure for males $\left(F_{(1,120)}=\right.$ $38.181, p<0.001)$. Error bars, SEM.

analyses revealed simple main effects of drug exposure for males in all of the above bursting characteristics $\left(F_{(1,108)}=9.465, p=\right.$ $0.003 ; F_{(1,108)}=33.382, p<0.001 ; F_{(1,108)}=29.746, p<0.001$; and $F_{(1,108)}=36.306, p<0.001$, respectively).

\section{Increased TH immunoreactivity within the LC and in neocortical noradrenergic fibers}

CTM-exposed male rats exhibited an increase in TH immunostaining intensity within the LC compared to saline exposure $\left(t_{(6)}=\right.$ 3.026, $p=0.023$ ), but this difference was not observed in females $\left(t_{(4)}=0.422, p=0.694\right.$ ) (Fig. 3). Although a trend was noticed in males, no significant change in TH immunostaining density within the LC was observed between male CTM and saline exposure $\left(t_{(6)}=\right.$ 1.573, $p=0.167)$ or between female CTM and saline exposure $\left(t_{(4)}=1.292, p=0.266\right)$.

TH-positive fibers are typically found in the dopaminergic axons within the striatum and limbic cortical areas such as medial prefrontal cortex, but expression is often too low to be detected in the neocortex (Pickel et al., 1975; Hökfelt et al., 1977). As expected, very few TH-positive fibers were observed in saline-exposed animals. In contrast, numerous $\mathrm{TH}$-positive fibers were found in the neocortex of CTMexposed male animals (Fig. 4). To determine whether these $\mathrm{TH}$-positive fibers are noradrenergic in origin, double immunofluorescent procedures were used, which revealed that virtually every $\mathrm{TH}$-positive fiber in the neocortex also expressed DBH and/or NET (Fig. 4). Furthermore, an approximately fourfold increase in noradrenergic $\mathrm{TH}$-positive fiber density was found in the neocortex of male CTMexposed animals compared to saline exposure $\left(t_{(6)}=1.961, p=0.098\right)$, while this trend was not seen in females $\left(t_{(4)}=\right.$ $0.200, p=0.851)$. Because the increased density of noradrenergic $\mathrm{TH}$-expressing neocortical fibers could be explained by an increased number of DBH-positive fibers, we analyzed the percentage change of DBH-positive fiber density and found no significant change in $\mathrm{DBH}$ expression in male CTM animals compared to saline $\left(t_{(5)}=0.008, p=0.994\right)$ or female CTM animals compared to saline $\left(t_{(4)}=0.344\right.$, $p=0.748)$.

\section{Discussion}

We conducted both electrophysiological and immunohistochemical techniques from the same set of animals to elucidate the neurodevelopmental effects of perinatal SSRI exposure on NE-LC circuit function. We demonstrated that brief neonatal exposure to one of the most selective SSRIs (CTM) created long-lasting changes to the NE-LC system including a hyperexcitability of single LC neurons and increased expression of $\mathrm{TH}$ in both the $\mathrm{LC}$ region and their neocortical targets. Furthermore, male rats appeared to be more vulnerable to this early monoamine system manipulation, suggesting a sex-specific response to early manipulation of the monoamine system. Since our previous studies have demonstrated that later exposure (PN8-PN21) to a smaller quantity of CTM $(10 \mathrm{mg} / \mathrm{kg} / \mathrm{d})$ downregulated 5-HT-raphe circuit function (Maciag et al., 2006; Weaver et al., 2010), and it is well known that 5-HT and NE can exert inverse modulatory actions (for review, see Berridge and Waterhouse, 2003), it is not surprising that the NE-LC system is upregulated. In brief, this study provided the first demonstration of a sexually dimorphic enhancement of the NE-LC system after perinatal exposure to antidepressants such as CTM. Our novel findings could have major implications for prescribing SSRIs to both children and pregnant and nursing mothers, and they provide further support for the theory of altered monoamine levels during critical periods of neurodevelopment as a possible etiology of pervasive developmental disorders such as ASD. 

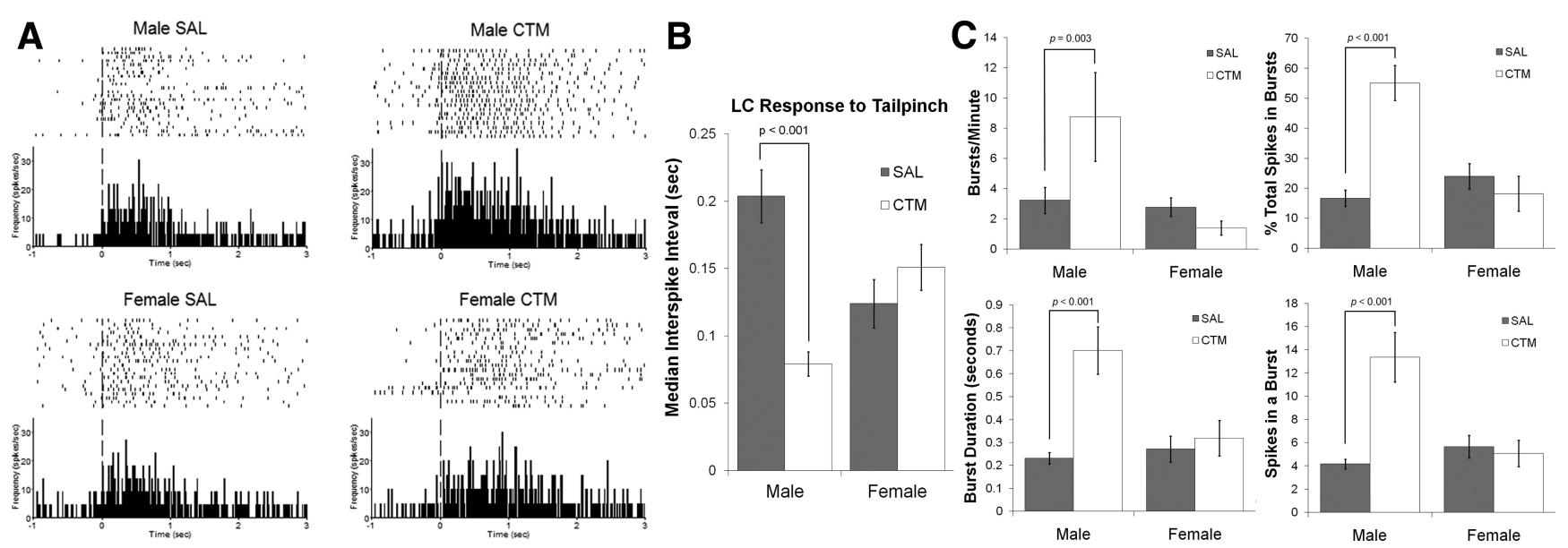

Figure 2. Tail-pinch elicited firing of single $L C$ neurons. $A, B$, The median interspike interval following tail-pinch was shorter (demonstrating faster firing) in male rats neonatally exposed to CTM $(0.079 \pm 0.035 \mathrm{~s} ; n=15)$ compared to male saline $(S A L)(0.204 \pm 0.135 \mathrm{sD} ; n=47)$, female SAL $(0.124 \pm 0.111 \mathrm{sSD} ; n=38)$, and female $\mathrm{CTM}(0.147 \pm 0.102 \mathrm{sSD} ; n=12)$ animals, verified with a significant sex by drug exposure interaction $\left(F_{(1,108)}=8.83, p=0.004\right)$ and a simple main effect of drug exposure for males $\left(F_{(1,108)}=14.052, p<0.001\right)$. Perievent rasters and corresponding histograms are of representative animals with the vertical dashed line illustrating the onset of tail-pinch. The raster plots illustrate the trial-by-trial responses of a single $L C$ neuron to tail-pinch. Histograms are in $10 \mathrm{~ms}$ bins converted to spikes/s to show the average response of the cell over $\sim 5 \mathrm{~min}$. C, Burst analysis of single $L($ neurons demonstrated a significant increase in (1) the number of bursts per minute, (2) the percentage of total spikes categorized within a burst, (3) mean burst duration, and (4) the number of spikes within a burst of male rats exposed to CTM compared to all other groups. Error bars, SEM.
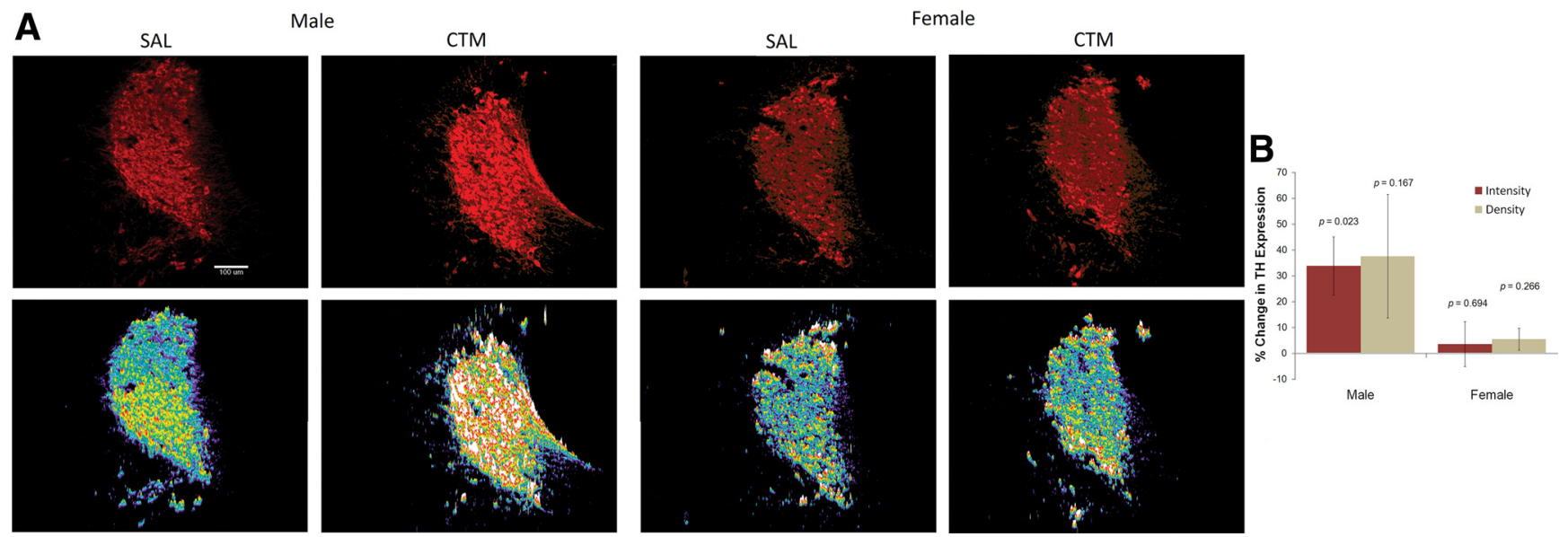

Figure 3. Immunoreactive expression of TH in the LC. $A$, The intensity of TH expression in the LC of male rats exposed to CTM increased compared to all other groups as seen in the photos of $C y 3$ (red)-labeled $\mathrm{LC}$ neurons (top) and in the corresponding intensity profiles (bottom). $\boldsymbol{B}$, The data were analyzed using a threshold measurement in specified regions containing $\mathrm{LC}$ neurons to determine the density (defined by percentage threshold area) and intensity (defined by average intensity of threshold area) of immunostaining. Histograms represent a percentage increase/ decrease in TH expression within the LC of (TM animals compared to their matched saline (SAL) controls that were stained in sets. (TM-exposed male rats exhibited a significant increase in TH immunostaining intensity within the $\mathrm{LC}$ compared to saline exposure $\left(t_{(6)}=3.026, p=0.023\right)$. Scale bar, $100 \mu \mathrm{m}$. Male sets $n=7$ and female sets $n=5$. Error bars, SEM.

Manipulating the 5-HT system during early neurodevelopment by exposure to SSRIs or through genetic means produces long-lasting changes in behavior as well as in the neurobiology of the 5-HT system (Mirmiran et al., 1981; Parks et al., 1998; Shih et al., 1999; Ansorge et al., 2004; Maciag et al., 2006; Oberlander et al., 2009; Homberg et al., 2010; Iñiguez et al., 2010; Weaver et al., 2010; Rodriguez-Porcel et al., 2011), but little is known about how this affects the adult NE-LC system [although blocking the NE transporter during neurodevelopment does not produce similar alterations in behavior (Ansorge et al., 2008)]. As we stated earlier, virtually all studies aimed at understanding the effects of SSRIs on the NE-LC system have been reported using mature animals (Nestler et al., 1990; Szabo et al., 1999; West et al., 2009). The overall conclusion derived from those studies is that such exposure in adults, especially chronic administration, leads to a reduction in LC firing rates and a downregulation of LC neural markers (e.g., TH). In contrast, West et al. (2010) reported that paroxetine-induced increases of LC activity in adolescent rats (PN45) were restricted to acute exposure (2-4 d), but were more similar to adult exposure (decreased LC activity) after longerterm treatment $(8-14 \mathrm{~d})$. This increased activity complements our results, but with the apparent difference in chronic versus acute exposure in the above-mentioned study, it is apparent that the identification of critical windows of antidepressant sensitivity on neurodevelopment needs to be investigated further. Nonetheless, it is clear that perinatal antidepressant exposure leads to changes in LC activity and these effects persist into adulthood, even after treatment has stopped.

There are continuously evolving theories of NE-LC circuit function that have begun to identify the specific contributions of the tonic and phasic firing repertories of single LC neurons in various contexts. These theories of NE-LC function include adaptive gain modes that operate on cortical circuits to optimize behavioral responses in familiar tasks (exploitation, phasic mode) or to search for 
A
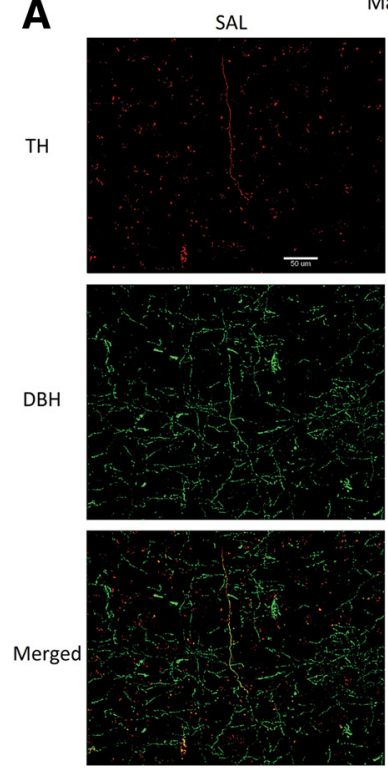

Male
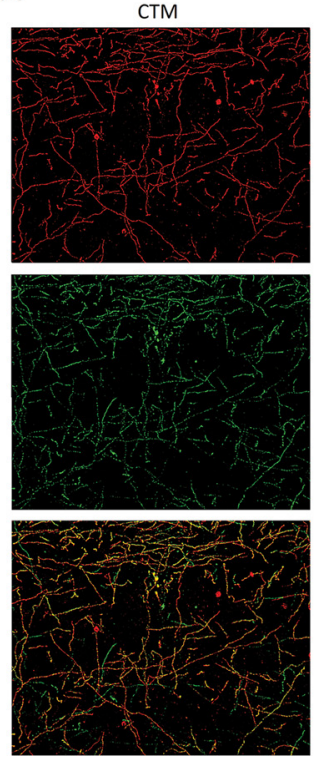
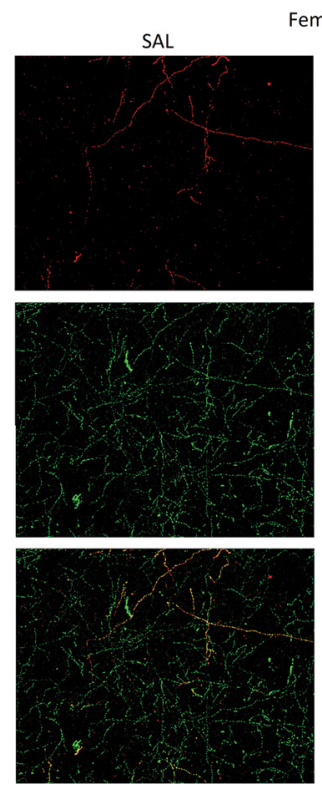

Female
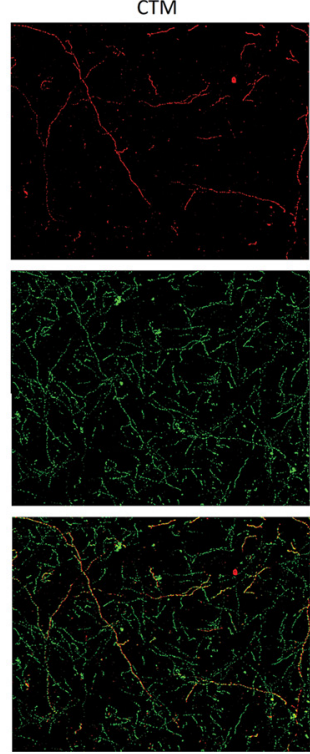

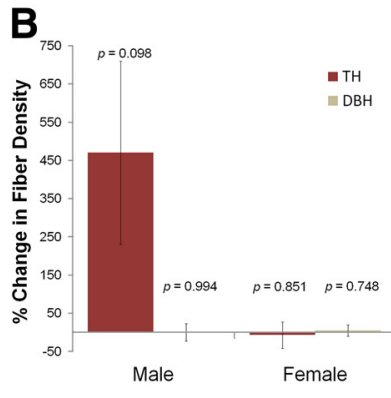

Figure 4. Immunoreactive expression of TH and DBH in the neocortex. $A$, The density of TH-positive fibers in the neocortex of male rats exposed to CTM increased as demonstrated in the photos of Cy3 (red)-labeled TH fibers (top). To determine a possible source of these fibers, the tissue was double stained with DBH (or NET) as shown in the photos of (y2 (green)-labeled DBH fibers (middle). Almost all TH-positive fibers were double labeled with DBH (merged-bottom), suggesting that the TH-positive fibers likely originated from the LC, and the increased TH expression may be due to the increased activity of the $L C$ neurons. $B$, The data were analyzed using a threshold measurement to determine the density (defined by percentage threshold area) of both TH-positive and DBH-positive immunostained fibers. Histograms represent a percentage increase/decrease in TH or DBH expression in CTM animals compared to their matched saline (SAL) controls that were stained in sets. CTM-exposed male rats exhibited an approximately fourfold increase in noradrenergic TH-positive fiber density in the neocortex compared to saline exposure $\left(t_{(6)}=1.961, p=0.098\right)$. Scale bar, $50 \mu \mathrm{m}$. Male sets $n=7$ and female sets $n=5$. Error bars, SEM.

new behaviors to determine optimal responses (exploration, tonic mode) (Aston-Jones and Cohen, 2005). Other theories focus more on arousal where the different modes serve to alter responses of sensory systems for amplification of perithreshold sensory stimuli (tonic mode) or to allow for the detection of more salient or novel subthreshold stimuli that would otherwise go undetected (phasic mode) (Berridge and Waterhouse, 2003; Devilbiss and Waterhouse, 2011). Therefore, changes in LC physiology could lead to altered behavioral flexibility and responding to salient environmental cues.

Our present data with perinatally CTM-exposed male rats revealed that the tonic firing rate of LC neurons was higher than in controls, and exhibited more and longer bursts than in male saline controls and females. Intriguingly, Usher et al. (1999) reported that poor performance of monkeys in a visual discrimination task was correlated with faster tonic LC activity $(\sim 3 \mathrm{~Hz})$, whereas better performance was correlated with slower activity $(\sim 2 \mathrm{~Hz})$, suggesting that higher tonic LC activity may be detrimental to behavioral performance when behavioral flexibility (i.e., increasing responsiveness to unexpected or novel stimuli) is preferred over heightened selectivity (e.g., in stable and predictable environments). This is complementary to our recent behavioral studies that have shown that perinatally CTM-exposed male rats exhibited neophobic-like behavior (Rodriguez-Porcel et al., 2011), suggesting the neophobia may be a result of altered NE-LC circuit function in response to novelty.

The effects of antidepressant exposure on the adult NE-LC circuit typically result in a reduction of LC firing rates with a corresponding decrease in expression of the catalyzing catecholamine enzyme tyrosine hydroxylase (TH) within the LC (Nestler et al., 1990; West et al., 2009). Decreased TH expression in the rat LC was accompanied by a corresponding decrease in mRNA levels, supporting the view that changes in LC neuronal firing corresponded to changes in protein and message expression within the LC (Nestler et al., 1990). It has also been established that stimulation of the LC results in enhanced NE release within their cortical target sites (Devoto et al., 2005), and this effect is greatest with burst stimulation (Florin-Lechner et al., 1996). The data presented here support this relationship between neuronal firing and the expression of TH within the LC and their cortical targets by demonstrating that perinatal exposure to SSRIs can increase the expression of TH in the NE-LC circuitry with corresponding increases in neuronal firing. The hyperexcitability of LC neurons with an increase in phasic bursting could be the mechanism responsible for the observable levels of TH within NE neocortical terminals, which typically are too weak to detect (Pickel et al., 1975; Hökfelt et al., 1977).

ASD has a sexual bias, with males affected four times more often than females (Zahn-Waxler et al., 2008). The precise mechanism(s) of the apparent sexual dimorphism for developing ASD is unknown, but abnormally high levels of 5-HT during critical periods of neurodevelopment has been suggested as a major contributing factor (Whitaker-Azmitia, 2005). For example, it has been reported that human males have higher rates of 5-HT synthesis (Nishizawa et al., 1997), and abnormal 5-HT synthesis during development is correlated with ASD (Chugani et al., 1999; Chandana et al., 2005). Thus, it is of interest that studies have demonstrated a sexual dimorphism in 5-HT receptor-mediated behavior and 5-HT levels (Jones and Lucki, 2005), LC development (Pinos et al., 2001), cortical monoamine levels (Connell et al., 2004), LC response to stress and corticotrophin-releasing factor (Curtis et al., 2006), and the sexual differentiation of monoaminergic neurons (Reisert and Pilgrim, 1991), all supporting a possible mechanism for a sexually dimorphic response to altered 5-HT levels during critical periods of neurodevelopment. To our knowledge, the present findings provide the first indication that perinatal exposure to CTM preferentially affects NE-LC circuit 
function in males, but the precise biological function for such sexually specific responses remains to be elucidated.

In animal models of depression, LC neural activity increases (Simson and Weiss, 1988), and SSRI exposure reduces this effect (West et al., 2009). Increased LC firing when SSRIs were administered during adolescence (PN45) was interpreted as a possible explanation for the increased risk for suicide in younger populations taking SSRIs (Goodman et al., 2007; West et al., 2010). We extend these findings to earlier periods of neurodevelopment and suggest that this earlier manipulation of the monoamine system may result in long-lasting modifications of the NE-LC system in males. Since perinatal antidepressant exposure alters both the 5-HT-raphe and NE-LC systems in adults, and both 5-HT and NE have been implicated in depression, it may be premature to suggest which specific system plays the key role for such dysfunction, and in fact, it may be a combination of the overall tone from both 5-HT and NE that contributes to the normal development of the monoamine system, their neocortical sites, their firing properties, and corresponding behaviors. Finally, our data are consistent with others who suggest that more research needs to be done regarding the safety and/or side effects of antidepressant prescriptions to children and pregnant or nursing mothers due to the potential adverse health consequences and the dramatic increase in prescription rates to these populations (Homberg et al., 2010; Kendall-Tackett et al., 2010; Croen et al., 2011).

\section{References}

Ansorge MS, Zhou M, Lira A, Hen R, Gingrich JA (2004) Early-life blockade of the 5-HT transporter alters emotional behavior in adult mice. Science 306:879-881.

Ansorge MS, Morelli E, Gingrich JA (2008) Inhibition of serotonin but not norepinephrine transport during development produces delayed, persistent perturbations of emotional behaviors in mice. J Neurosci 28:199-207.

Aston-Jones G, Bloom FE (1981) Norepinephrine-containing locus coeruleus neurons in behaving rats exhibit pronounced responses to nonnoxious environmental stimuli. J Neurosci 1:887-900.

Aston-Jones G, Cohen JD (2005) An integrative theory of locus coeruleusnorepinephrine function: adaptive gain and optimal performance. Annu Rev Neurosci 28:403-450.

Berridge CW, Waterhouse BD (2003) The locus coeruleus-noradrenergic system: modulation of behavioral state and state-dependent cognitive processes. Brain Res Rev 42:33-84.

Bobker DH, Williams JT (1989) Serotonin agonists inhibit synaptic potentials in the rat locus ceruleus in vitro via 5-hydroxytryptamine $1 \mathrm{~A}$ and 5-hydroxytryptamine 1B receptors. J Pharmacol Exp Ther 250:37-43.

Casper RC, Fleisher BE, Lee-Ancajas JC, Gilles A, Gaylor E, DeBattista A, Hoyme HE (2003) Follow-up of children of depressed mothers exposed or not exposed to antidepressant drugs during pregnancy. J Pediatr 142:402-408

Cedarbaum JM, Aghajanian GK (1978) Afferent projections o the rat locus coeruleus as determined by a retrograde tracing technique. J Comp Neurol 178:1-16.

Chandana SR, Behen ME, Juhász C, Muzik O, Rothermel RD, Mangner TJ, Chakraborty PK, Chugani HT, Chugani DC (2005) Significance of abnormalities in developmental trajectory and asymmetry of cortical serotonin synthesis in autism. Int J Dev Neurosci 23:171-182.

Chugani DC, Muzik O, Behen M, Rothermel R, Janisse JJ, Lee J, Chugani HT (1999) Developmental changes in brain serotonin synthesis capacity in autistic and nonautistic children. Ann Neurol 45:287-295.

Cohen D (2007) Should the use of selective serotonin reuptake inhibitors in child and adolescent depression be banned? Psychotherapy and Psychosomatics 76:5-14.

Connell S, Karikari C, Hohmann CF (2004) Sex-specific development of cortical monoamine levels in mouse. Brain Res Dev Brain Res 151:187-191.

Croen LA, Grether JK, Yoshida CK, Odouli R, Hendrick V (2011) Antidepressant use during pregnancy and childhood spectrum disor- ders. Arch Gen Psychiatry. Advance online publication. Retrieved September 23, 2011. doi:10.1001/archgenpsychiatry.2011.73.

Curtis AL, Bethea T, Valentino RJ (2006) Sexually dimorphic responses of the brain norepinephrine system to stress and corticotrophin-releasing factor. Neuropsychopharmacology 31:544-554.

Devilbiss DM, Waterhouse BD (2011) Phasic and tonic patterns of locus coeruleus output differentially modulate sensory network function in the awake rat. J Neurophysiol 105:69-87.

Devoto P, Flore G, Saba P, Fà M, Gessa GL (2005) Stimulation of the locus coeruleus elicits noradrenaline and dopamine release in the medial prefrontal and parietal cortex. J Neurochem 92:368-374.

Florin-Lechner SM, Druhan JP, Aston-Jones G, Valentino RJ (1996) Enhanced norepinephrine release in prefrontal cortex with burst stimulation of the locus coeruleus. Brain Res 742:89-97.

Gaspar P, Cases O, Maroteaux L (2003) The developmental role of serotonin: news from mouse molecular genetics. Nat Rev Neurosci 4: 1002-1012.

Goodman WK, Murphy TK, Storch EA (2007) Risk of adverse behavioral effects with pediatric use of antidepressants. Psychopharmacology 191: 87-96.

Haddjeri N, de Montigny C, Blier P (1997) Modulation of the firing activity of noradrenergic neurons in the rat locus coeruleus by the 5hydroxytryptamine system. Br J Pharmacol 120:865-875.

Hendrick V, Smith LM, Suri R, Hwang S, Haynes D, Altshuler L (2003) Birth outcomes after prenatal exposure to antidepressant medication. Am J Obstet Gynecol 188:812-815.

Hökfelt T, Johansson O, Fuxe K, Goldstein M, Park D (1977) Immunohistochemical studies on the localization and distribution of monoamine neuron systems in the rat brain II. Tyrosine hydroxylase in the telencephalon. Med Biol 55:21-40.

Homberg JR, Schubert D, Gaspar P (2010) New perspectives on the neurodevelopmental effects of SSRIs. Trends Pharmacol Sci 31:60-65.

Iñiguez SD, Warren BL, Bolaños-Guzmán CA (2010) Short-and long-term functional consequences of fluoxetine exposure during adolescence in male rats. Biol Psychiatry 67:1057-1066.

Jones MD, Lucki I (2005) Sex differences in the regulation of serotonergic transmission and behavior in 5-HT receptor knockout mice. Neuropsychopharmacology 30:1039-1047.

Kendall-Tackett K, Hale TW (2010) The use of antidepressants in pregnant and breastfeeding women: a review of recent studies. J Hum Lact 26:187-195.

Kim MA, Lee HS, Lee BY, Waterhouse BD (2004) Reciprocal connections between subdivisions of the dorsal raphe and the nuclear core of the locus coeruleus in the rat. Brain Res 1026:56-67.

Luppi PH, Aston-Jones G, Akaoka H, Chouvet G, Jouvet M (1995) Afferent projections to the rat locus coeruleus demonstrated by retrograde and anterograde tracing with cholera-toxin B subunit and Phaseolus vulgaris leucoagglutinin. Neuroscience 65:119-160.

Maciag D, Simpson KL, Coppinger D, Lu Y, Wang Y, Lin RCS, Paul IA (2006) Neonatal antidepressant exposure has lasting effects on behavior and serotonin circuitry. Neuropsychopharmacology 31:47-57.

McRae-Degueurce A, Berod A, Mermet A, Keller A, Chouvet G, Joh TH, Pujol JF (1982) Alterations in tyrosine hydroxylase activity elicited by raphe nuclei lesions in the rat locus coeruleus: evidence for the involvement of serotonin afferents. Brain Res 235:285-301.

Mirmiran M, Van De Poll NE, Corner MA, Van Oyen HG, Bour HL (1981) Suppression of active sleep by chronic treatment with chlorimipramine during early postnatal development: effects upon adult sleep and behavior in the rat. Brain Res 204:129-146.

Moses-Kolko EL, Bogen D, Perel J, Bregar A, Uhl K, Levin B, Wisner KL (2005) Neonatal signs after late in utero exposure to serotonin reuptake inhibitors: literature review and implications for clinical applications. JAMA 293:2372-2383.

Nestler EJ, McMahon A, Sabban EL, Tallman JF, Duman RS (1990) Chronic antidepressant administration decreases the expression of tyrosine hydroxylase in the rat locus coeruleus. Proc Natl Acad Sci U S A 87:7522-7526.

Nishizawa S, Benkelfat C, Young SN, Leyton M, Mzengeza S, de Montigny C, Blier P, Diksic M (1997) Differences between males and females in rates of serotonin synthesis in human brain. Proc Natl Acad Sci U S A 94:5308-5313.

Oberlander TF, Gingrich JA, Ansorge MS (2009) Sustained neurobehav- 
ioral effects of exposure to SSRI antidepressants during development: molecular to clinical evidence. Clin Pharmacol Ther 86:672-677.

Parks CL, Robinson PS, Sibille E, Shenk T, Toth M (1998) Increased anxiety of mice lacking the serotonin1A receptor. Proc Natl Acad Sci U S A 95:10734-10739.

Paxinos G, Watson C (1986) The rat brain in stereotaxic coordinates, 2nd edition. New York: Academic.

Pickel VM, Joh TH, Reis DJ (1975) Ultrastructural localization of tyrosine hydroxylase in noradrenergic neurons of brain. Proc Natl Acad Sci U S A 72:659-663.

Pinos H, Collado P, Rodríguez-Zafra M, Rodríguez C, Segovia S, Guillamón A (2001) The development of sex differences in the locus coeruleus of the rat. Brain Res Bull 56:73-78.

Reisert I, Pilgrim C (1991) Sexual differentiation of monaminergic neurons-genetic or epigenetic. Trends Neurosci 14:468-473.

Rodriguez-Porcel F, Green D, Khatri N, Harris SS, May WL, Lin RCS, Paul IA (2011) Neonatal exposure of rats to antidepressants affects behavioral reactions to novelty and social interactions in a manner analogous to autistic spectrum disorders. Anat Rec 294:1726-1735.

Sanders JD, Happe HK, Bylund DB, Murrin LC (2005) Development of the norepinephrine transporter in the rat CNS. Neuroscience 130:107-117.

Seager MA, Huff KD, Barth VN, Phebus LA, Rasmussen K (2004) Fluoxetine administration potentiates the effect of olanzapine on locus coeruleus neuronal activity. Biol Psychiatry 55:1103-1109.

Shih JC, Chen K, Ridd MJ (1999) Monoamine oxidase: from genes to behavior. Annu Rev Neurosci 22:197-217.

Simson PE, Weiss JM (1988) Altered activity of the locus coeruleus in an animal model of depression. Neuropsychopharmacology 1:287-295.
Szabo ST, de Montigny C, Blier P (1999) Modulation of noradrenergic neuronal firing by selective serotonin reuptake blockers. Br J Pharmacol 126:568-571.

Usher M, Cohen JD, Servan-Schreiber D, Rajkowski J, Aston-Jones G (1999) The role of locus coeruleus in the regulation of cognitive performance. Science 283:549-554.

Weaver KJ, Paul IA, Lin RCS, Simpson KL (2010) Neonatal exposure to citalopram selectively alters the expression of the serotonin transporter in the hippocampus: dose-dependent effects. Anat Rec (Hoboken) 293:1920-1932

West CHK, Ritchie JC, Boss-Williams KA, Weiss JM (2009) Antidepressant drugs with differing pharmacological actions decrease activity on locus coeruleus neurons. Int J Neuropsychopharmacol 12:627-641.

West CHK, Ritchie JC, Weiss JM (2010) Paroxetine-induced increase in activity of locus coeruleus neurons in adolescent rats: implication of a countertherapeutic effect of an antidepressant. Neuropsychopharmacology 35:1653-1663.

Whitaker-Azmitia PM (2005) Behavioral and cellular consequences of increasing serotonergic activity during brain development: a role in autism. Int J Dev Neurosci 23:75-83.

Zahn-Waxler C, Shirtcliff EA, Marceau K (2008) Disorders of childhood and adolescence: gender and psychopathology. Annu Rev Clin Psychol 4:275-303.

Zhang J, Darling RD, Paul IA, Simpson KL, Chen K, Shih JC, Lin RCS (2011) Altered expression of tyrosine hydroxylase in the locus coeruleus noradrenergic system in citalopram neonatally exposed rats and monoamine oxidase A knock out mice. Anat Rec (Hoboken) 294:1685-1697. 